STRUCTURAL SCIENCE CRYSTAL ENGINEERING MATERIALS

ISSN 2052-5206

\section{Transition Metal Compounds. By Daniel I. Khomskii. Cambridge University Press, 2014. Pp. 496. Price GBP 80.00, USD 125.00. ISBN 9781107020177.}

Keywords: book review; transition metals; solidstate research.

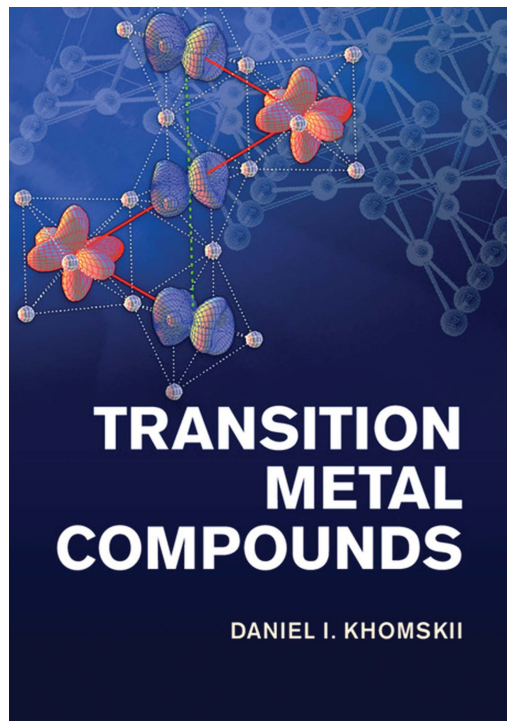

(C) 2016 International Union of Crystallography

\author{
Bernhard Keimer*
}

Max-Planck-Institute for Solid State Research, Heisenbergstr. 1, D-70569 Stuttgart, Germany. *Correspondence e-mail: b.keimer@fkf.mpg.de

Following the discovery of spectacular phenomena such as high-temperature superconductivity and colossal magnetoresistance, transition metal compounds have been at the forefront of solid-state research for the past three decades. The research inspired by these discoveries is extremely diverse, ranging from fundamental studies of model Hamiltonians with strong electronic correlations all the way to the synthesis and characterization of next-generation electronic devices. The result is a massive body of knowledge that even insiders often find bewildering. In the course of a long and distinguished career, Daniel Khomskii has made leading contributions to many aspects of research on transition metal compounds. Due to his overview of the field and his pedagogical skill, he is much sought after as a lecturer at summer schools, and his typewritten lecture notes have been circulating among researchers for some time. The book lives up to this reputation and offers a comprehensive yet highly readable introduction to the field.

The introductory Chapter 1 exposes the dichotomy between the itinerant electronic states treated in standard textbooks on solid-state physics and the localized states that are required to describe the strongly correlated electron systems in transition metal compounds. It also introduces the Hubbard model and the concept of a Mott insulator. Chapter 2 summarizes basic concepts of atomic physics including Hund's rules and the spin-orbit coupling.

The next two chapters describe issues that are germane to transition metal compounds, starting from a description of the most common lattice architectures and the steric constraints that underlie their formation. Chapter 3 also outlines the interactions of transition metal ions with the surrounding lattice, including charge transfer between the ionic species and crystal fields acting on the transition metal ions, as well as their interplay with the intra-atomic interactions. Chapter 4 discusses the hybridization between transition metal and ligand states and its consequences for the basic electronic structure of transition metal compounds. These include issues such as the classification of insulating metal oxides into Mott Hubbard and charge transfer insulators and the formation of Zhang-Rice singlets, which are still very much debated in the field.

Chapters 5-8 address electronic ordering phenomena that are widely observed in transition metal compounds. Magnetic order and the exchange interactions driving its formation are topics of Chapter 5. Starting from a basic discussion of superexchange and double-exchange interactions, the subsequent discussion also covers issues of current interest such as geometrical frustration, collective singlet states and spin-state transitions. Chapter 6 describes orbital ordering in insulators and its impact on magnetism, moving on to the Kugel-Khomskii Hamiltonian and the quantum dynamics of orbitals that have been subjects of intense recent research.

Charge order and its interplay with orbital and magnetic order are reviewed in Chapter 7. Here as elsewhere, the author first discusses the key factors driving charge ordering in well known materials such as magnetite and manganese oxides, and then draws interesting parallels to other compounds such as silver and gold compounds that have been less widely studied. Chapter 8 covers ferroelectricity and its combination with different forms of collective order. The discussion of the different origins of multiferroicity, which builds on the understanding of spin, charge and orbital order carefully developed in the previous chapters, is one of the highlights of this book. 
Whereas the earlier chapters mostly deal with insulating, stoichiometric compounds, Chapter 9 contains various examples of lightly doped Mott insulators which host some of the most fascinating phenomena such as high-temperature superconductivity. Besides discussing superconductivity in copper oxides and iron pnictides and chalcogenides, the chapter also briefly touches on topical issues such as quantum criticality, electronic phase separation, and metal-oxide interfaces that are at the heart of novel field-effect devices. It is thus a lively guide to the current literature. Chapter 10 then moves on to fully developed metallic states and correlationdriven metal-insulator transitions, with a focus on classical examples such as $\mathrm{VO}_{2}$ and $\mathrm{V}_{2} \mathrm{O}_{3}$. The final Chapter 11 contrasts the $d$-electron systems encountered in transition metal compounds with the $f$-electron systems in rare earth and actinide compounds, and goes on to discuss the Kondo effect, the formation of local moments in metals, and the role of rareearth ions in transition metal compounds.
The book is carefully structured and very well written. The author explains the main physical ideas in each subject area in a transparent manner, without going into too much mathematical or technical detail, and he provides plenty of helpful illustrations. Since the physical intuition and understanding are developed systematically, and not every reader may be deeply interested in every subject area, a concise summary is provided at the end of each chapter. The book will thus be a great resource for students entering the field and for seasoned researchers alike, and it will be of lasting value as the field continues its rapid expansion. If I had one wish free for a future edition, it would be for a chapter on ion transport, which is becoming increasingly important in view of applications of transition metal compounds in batteries and fuel cells.

The book is currently only available in hardcover format and comes at a rather steep price, but I highly recommend this investment to anyone who is seriously interested in this fascinating area of research. 